\title{
Review
}

Neuro

epidemiology

\section{The Epidemiology of Epilepsy}

\author{
Ettore Beghi \\ Department of Neuroscience, Istituto di Ricerche Farmacologiche Mario Negri IRCCS, Milan, Italy
}

\section{Keywords}

Epilepsy $\cdot$ Incidence $\cdot$ Prevalence $\cdot$ Mortality $\cdot$ Burden

\begin{abstract}
Epilepsy is a chronic disease of the brain characterized by an enduring (i.e., persisting) predisposition to generate seizures, unprovoked by any immediate central nervous system insult, and by the neurobiologic, cognitive, psychologi$\mathrm{cal}$, and social consequences of seizure recurrences. Epilepsy affects both sexes and all ages with worldwide distribution. The prevalence and the incidence of epilepsy are slightly higher in men compared to women and tend to peak in the elderly, reflecting the higher frequency of stroke, neurodegenerative diseases, and tumors in this age-group. Focal seizures are more common than generalized seizures both in children and in adults. The etiology of epilepsy varies according to the sociodemographic characteristics of the affected populations and the extent of the diagnostic workup, but a documented cause is still lacking in about $50 \%$ of cases from high-income countries (HIC). The overall prognosis of epilepsy is favorable in the majority of patients when measured by seizure freedom. Reports from low/middle-income countries (LMIC; where patients with epilepsy are largely untreated) give prevalence and remission rates that overlap those of HICs. As the incidence of epilepsy appears higher in most LMICs, the overlapping prevalence can be explained by misdiagnosis, acute symptomatic seizures and premature mortality. Studies have consistently shown that about one-half of cases tend to achieve prolonged seizure remission. However, more recent reports on the long-term prognosis of ep-
\end{abstract}

ilepsy have identified differing prognostic patterns, including early and late remission, a relapsing-remitting course, and even a worsening course (characterized by remission followed by relapse and unremitting seizures). Epilepsy per se carries a low mortality risk, but significant differences in mortality rates are expected when comparing incidence and prevalence studies, children and adults, and persons with idiopathic and symptomatic seizures. Sudden unexplained death is most frequent in people with generalized tonicclonic seizures, nocturnal seizures, and drug refractory epilepsy.

(c) 2019 S. Karger AG, Base

Epilepsy is one of the most common neurological diseases and affects people of all ages, races, social classes, and geographical locations. Epilepsy is a disease of the brain characterized by an enduring predisposition to generate seizures and by the neurobiologic, cognitive, psychological, and social consequences of seizure recurrences [1]. The epileptic seizures are recurrent paroxysmal events characterized by stereotyped behavioral alterations that reflect the underlying neural mechanisms of the disease. The differential diagnosis of epilepsy encompasses a number of clinical conditions characterized by transient alteration of awareness and/or behavior. In most cases, the disease can be diagnosed through a careful history or by the observation of a seizure. Although an etiologic agent can be identified, still in about one half of cases, the cause is unknown [2]. A variable genetic predisposition to manifest seizures and the differing distribution of some environmental risk factors can explain the het-

\section{KARGER}

(c) 2019 S. Karger AG, Basel

karger@karger.com

www.karger.com/ned
Ettore Beghi, MD

Department of Neuroscience, Laboratory of Neurological Disorders Istituto di Ricerche Farmacologiche Mario Negri IRCCS

Via Giuseppe La Masa 19, IT-20156 Milan (Italy)

E-Mail ettore.beghi@ marionegri.it 
erogeneity of the frequency, course, and consequences of the disease in the world. In addition to the recurrence of seizures, the underlying cause and the adverse effects of treatment have neurologic, cognitive, psychological, and social consequences that significantly affect the quality of life of the affected individuals and make the disease a complex nosographic entity.

\section{Definitions and Terminology}

While all people with epilepsy experience seizures, not all individuals with seizures have epilepsy. Epileptic seizures may also occur after an acute central nervous system (CNS) insult (structural, systemic, toxic, or metabolic). These events (acute symptomatic or provoked seizures) are intended as acute manifestations of the insult [3] and may not recur when the underlying cause has been removed or the acute phase has elapsed [4]. According to the International League Against Epilepsy (ILAE), epilepsy is defined by any of the following conditions: (1) at least 2 unprovoked (or reflex) seizures occurring $>24 \mathrm{~h}$ apart; (2) one unprovoked (or reflex) seizure and a probability of further seizures similar to the general recurrence risk (at least $60 \%$ ) after 2 unprovoked seizures, occurring over the next 10 years; and (3) diagnosis of an epilepsy syndrome [5]. However, for the purpose of conducting population-based studies, the ILAE Epidemiology Commission advises that epilepsy be defined as 2 or more unprovoked seizures occurring at least $24 \mathrm{~h}$ apart [6].

An unprovoked seizure is a seizure occurring in the absence of precipitating factors. Unprovoked seizures include events occurring in the absence of recognized etiological or risk factors (idiopathic and cryptogenic seizures), in patients with antecedent stable (nonprogressing) CNS insults (remote symptomatic seizures), or in those with progressive CNS abnormalities, like brain tumors or degenerative conditions (progressive symptomatic seizures).

Seizure onset can be focal (seizures arising in one hemisphere of the brain), generalized (seizures originating in both hemispheres simultaneously), and unknown [7]. Focal seizures are classified according to whether awareness (a marker for consciousness) is intact or impaired. Focal and generalized seizures are also divided into motor and nonmotor.

Active epilepsy is defined by regular treatment with antiepileptic medications or when the most recent seizure has occurred within the last 5 years [6].

Status epilepticus (SE) is an epileptic seizure that is sufficiently prolonged or repeated at sufficiently brief inter- vals so as to produce an enduring epileptic condition. SE can have long-term consequences including neuronal injury or death and alteration of neuronal networks, depending on the type and duration of seizures. A new diagnostic classification of SE has been recently proposed [8].

Sudden unexpected death in epilepsy (SUDEP) is the sudden, unexpected, witnessed or unwitnessed, nontraumatic, and nondrowning death in patients with epilepsy, with or without evidence for a seizure and excluding documented SE, in which postmortem examination does not reveal a toxicologic or anatomic cause of death [9]. In most cases, SUDEP is triggered by a seizure, and seizureinduced cardiorespiratory alterations are a plausible hypothesis.

Measures of the frequency of epilepsy include incidence, prevalence, and mortality; measures of the burden of epilepsy are the disability-adjusted life-years (DALYs) and their components, the years of life lost, and the years of living with disability.

\section{Incidence of Acute Symptomatic Seizures}

The median incidence of acute symptomatic seizures is $29-39$ per 100,000 per year [10]. Acute symptomatic seizures predominate in the youngest age class (under 1 year of age) and in the elderly. Fever, traumatic brain injury (TBI), cerebrovascular disease, drug withdrawal, infection, and metabolic insults are the commonest precipitating factors.

\section{Incidence of Epilepsy}

In a systematic review and meta-analysis of incidence studies, the pooled incidence rate of epilepsy was 61.4 per 100,000 person-years (95\% CI 50.7-74.4) [11]. The incidence was higher in low/middle-income countries (LMIC) than in high-income countries (HIC), 139.0 (95\% CI 69.4-278.2) vs. 48.9 (95\% CI 39.0-61.1). This can be explained by the different structure of populations at risk and a greater exposure to perinatal risk factors, higher rates of CNS infections, and TBI in LMIC. The incidence of epilepsy is also higher in the lowest socioeconomic classes in HIC and, within the same population, people of differing ethnic origin [12]. Differences can be also explained by methodological issues, such as more stringent case verification and the exclusion of isolated and acute symptomatic seizures in some studies. 


\section{Prevalence of Epilepsy}

The prevalence of epilepsy differs significantly among countries depending on the local distribution of risk and etiologic factors, the number of seizures at diagnosis and if considering only active epilepsy (active prevalence) or including also cases in remission (lifetime prevalence). In the Fiest et al. [11], the overall lifetime prevalence of epilepsy was 7.60 per 1,000 population (95\% CI 6.17-9.38) and was higher in LMIC (8.75 per 1,000; 95\% CI 7.2310.59) than in HIC (5.18 per 1,000 ; $95 \%$ CI $3.75-7.15$ ). The point prevalence of active epilepsy was 6.38 per 1,000 (95\% CI 5.57-7.30). The median point prevalence of active epilepsy in LMIC was 6.68 (95\% CI 5.45-8.10) and in HIC was 5.49 (4.16-7.26). In selected populations, prevalence estimates also vary and tend to be higher in individuals of certain ethnicities [13], people in poor health, and socially deprived subjects [14]. Along with issues in the study design, the demographic structure of the study population, the prevalence of environmental risk factors, and the quality of health management can be implicated.

\section{Incidence and Prevalence of Epilepsy by Sex and Age}

Incidence and prevalence of epilepsy are slightly higher in men than in women [11]. The difference might be explained by the different prevalence of the most common risk factors and the concealment of the condition in women for sociocultural reasons in certain regions [15].

The incidence of epilepsy is higher in the youngest and oldest age-groups [11], with estimates of 86 per 100,000 per year in a well-defined population in the first year of age, a trend to decrease to about $23-31$ per 100,000 in people aged 30-59 years, and a subsequent increase up to 180 per 100,000 in the over 85 age-group [16]. In children, the incidence of epilepsy is highest in the first year of life and declines to adult levels by the end of 10 years of age [17]. In LMIC, epilepsy peaks in children; this may be a result of under-ascertainment of the condition in older individuals as well as the demographic structure of the country.

\section{Temporal Trends of Epilepsy}

In the last decades, the age-specific incidence of epilepsy has decreased with time in the youngest age-groups, probably due to improvements in perinatal care, better sanitation, and increased control of infectious diseases
[18]. In contrast, the incidence has increased in the elderly, likely due to improved life expectancy (with parallel increase of aging-related epileptogenic conditions, such as stroke, tumors, and neurodegenerative disorders) and increased ascertainment of the disease in this age-group.

\section{Incidence and Prevalence by Seizure Type}

Focal seizures are the predominant seizure type both in children and in adults $[16,19]$. The most common type of focal seizure is a focal impaired awareness seizure (accounting for approximately $36 \%$ of all people with seizures) [16]. In most LMIC, however, the predominant types reported are generalized tonic-clonic seizures [20], a reflection of under-ascertainment of the other seizure types, likely due to a lack of recognition and diagnostic tools. The incidence of SE has been found to vary from 6.8 to 41 per 100,000 per year [21] with a bimodal distribution (peaks in children $<1$ year and the elderly). The wide range can be explained by the population at risk, the accuracy of the diagnosis, the differing distribution of the underlying causes, and the inclusion or exclusion of acute symptomatic seizures.

\section{Incidence and Prevalence by Epilepsy Type}

In a population-based study done 20 years ago in a US population [22], focal epilepsies of unknown etiology were the most common group in people newly diagnosed with epilepsy ( 17.5 cases per 100,000 per year), followed by symptomatic partial epilepsies (focal epilepsies of structural or metabolic etiology according to the new ILAE classification) [23] (17.2), unknown epilepsies (epilepsies of unknown etiology; 9.7), symptomatic/cryptogenic epilepsies (epilepsies of structural or metabolic etiology/unknown etiology; 4.0), idiopathic generalized epilepsies (3.7), and idiopathic partial epilepsies (i.e., generalized and focal epilepsies of presumed genetic origin; 0.2 ). The proportion of epilepsies with unknown etiology has remained substantially unchanged in more recent years, at least in HIC [24].

In children, age at onset was significantly correlated with etiology. Approximately half had a documentable etiology. Of them, $28 \%$ were structural/metabolic, which predominated when seizures started before 12 months of age, and $22 \%$ were presumedly genetic, most likely associated with older age at onset [19]. A specific epilepsy syndrome could be detected in $28 \%$ of cases at first diagnosis. 


\section{Prognosis of Epilepsy}

Epilepsy is a treatable condition, with up to $80 \%$ entering prolonged periods of seizure remission and up to $50 \%$ continuing to be seizure-free after treatment discontinuation [25, 26]. However, reports from several LMIC (where treatment gap is high) give prevalence and remission rates overlapping to HIC [27]. As in most LMIC, the incidence of epilepsy is higher than in HIC and increased mortality can explain only in part the difference between incidence and prevalence, misdiagnosis and acute symptomatic seizures must be also considered.

Studies in newly diagnosed patients have consistently shown that $55-68 \%$ of cases tend to achieve prolonged seizure remission [27]. However, in a long-term population-based study done in patients with childhood-onset epilepsy, differing remission patterns were seen. Half of the patients entered terminal remission, without relapse, and one-fifth after relapse. About one-third had a poor outcome in terms of absolute absence of remission or relapsing seizures after periods of remission [26]. These patterns have been confirmed in part by others [28-31].

The risk of relapse after a first unprovoked seizure in population-based studies was fairly consistent with 36$37 \%$ rates at 1 year and $43-45 \%$ rates at 2 years [27]. In a systematic review, the average recurrence risk was $51 \%$ (95\% CI 49-53\%) [32]. After a first unprovoked seizure, the probability of a relapse decreases with time. About $50 \%$ of recurrences occur within 6 months. A documented etiology of the seizure and an abnormal (epileptiform and/or slow) electroencephalogram (EEG) pattern are the 2 most consistent predictors of recurrence. The pooled 2-year recurrence risk is lowest for an idiopathic or cryptogenic first seizure with a normal EEG (24\%; 95\% CI 19-29\%), intermediate for a remote symptomatic seizure (48\%; 95\% CI 34-62\%) with normal EEG or an idiopathic/cryptogenic seizure with an abnormal EEG (48\%; 95\% CI $40-55 \%$ ), and highest with a remote symptomatic seizure with an abnormal EEG (65\%; 95\% CI 55-76\%) [32]. Interictal EEG epileptiform abnormalities tend to be associated with a higher risk of seizure recurrence than non-epileptiform abnormalities. Seizures occurring during sleep are also associated with a higher risk of recurrence both in children and in adults. Focal seizures are also correlated with a higher risk of recurrence, even after controlling for etiology and EEG abnormalities. A positive correlation between seizure relapse and family history of seizures has been confirmed in patients with idiopathic or cryptogenic first seizures. History of acute symptomatic seizures prior to the first unprovoked sei- zure has been found to increase the risk of relapse, while evidence is inconclusive or lacking for sex, age, and SE.

The prognosis of untreated epilepsy can be assessed only in LMIC where epilepsy is largely untreated (treatment gap ranging from 70 to $94 \%$ ) [33]. In a populationbased study done in Ecuador, the cumulative annual incidence rate was 190 per 100,000 and the prevalence rate of active epilepsy was 7 per 1,000 , which implies a remission rate of at least $50 \%$ [34]. Similar prevalence rates of active epilepsy were found in other countries [35, 36]. These findings lend support to the hypothesis that spontaneous remission of epilepsy is a common event.

Etiology of epilepsy is the strongest prognostic predictor for seizure recurrence. In a well-defined US population, symptomatic epilepsies had a significantly lower chance of 5-year remission compared to idiopathic epilepsies (30 vs. $42 \%$ at 15 years) and patients with neurological dysfunction present at birth had the lowest chance of remission [25]. Other prognostic indicators included seizure type and EEG epileptiform abnormalities. Lower remission rates in patients with symptomatic epilepsies were found also in Europe [27].

As proposed by Sander [37], epilepsy patients can be classified into 4 different prognostic groups: (1) Excellent prognosis (about $20-30 \%$ of the total) with high probability of spontaneous remission; these include benign focal epilepsies, benign myoclonic epilepsy in infancy, and epilepsies provoked by specific modes of activation, that is, reflex epilepsies; (2) Good prognosis (about 30-40\%) with easy pharmacological control and possibility of spontaneous remission; these include childhood absence epilepsy and some focal epilepsies; (3) Uncertain prognosis (about 10-20\%), which may respond to drugs, but tend to relapse after treatment withdrawal; these include juvenile myoclonic epilepsy and most focal epilepsies (symptomatic or cryptogenic); (4) Poor prognosis (about $20 \%$ ) in which seizures tend to recur despite intensive treatment; these include epilepsies associated with congenital neurological defects, progressive neurological disorders, and some symptomatic or cryptogenic partial epilepsies. This classification is still valid even after the advent of more sophisticated diagnostic techniques and after the introduction of several new antiepileptic drugs.

\section{Mortality of Epilepsy}

Epilepsy per se carries a low mortality risk, but significant differences in mortality rates are expected when comparing incidence and prevalence studies, children and 


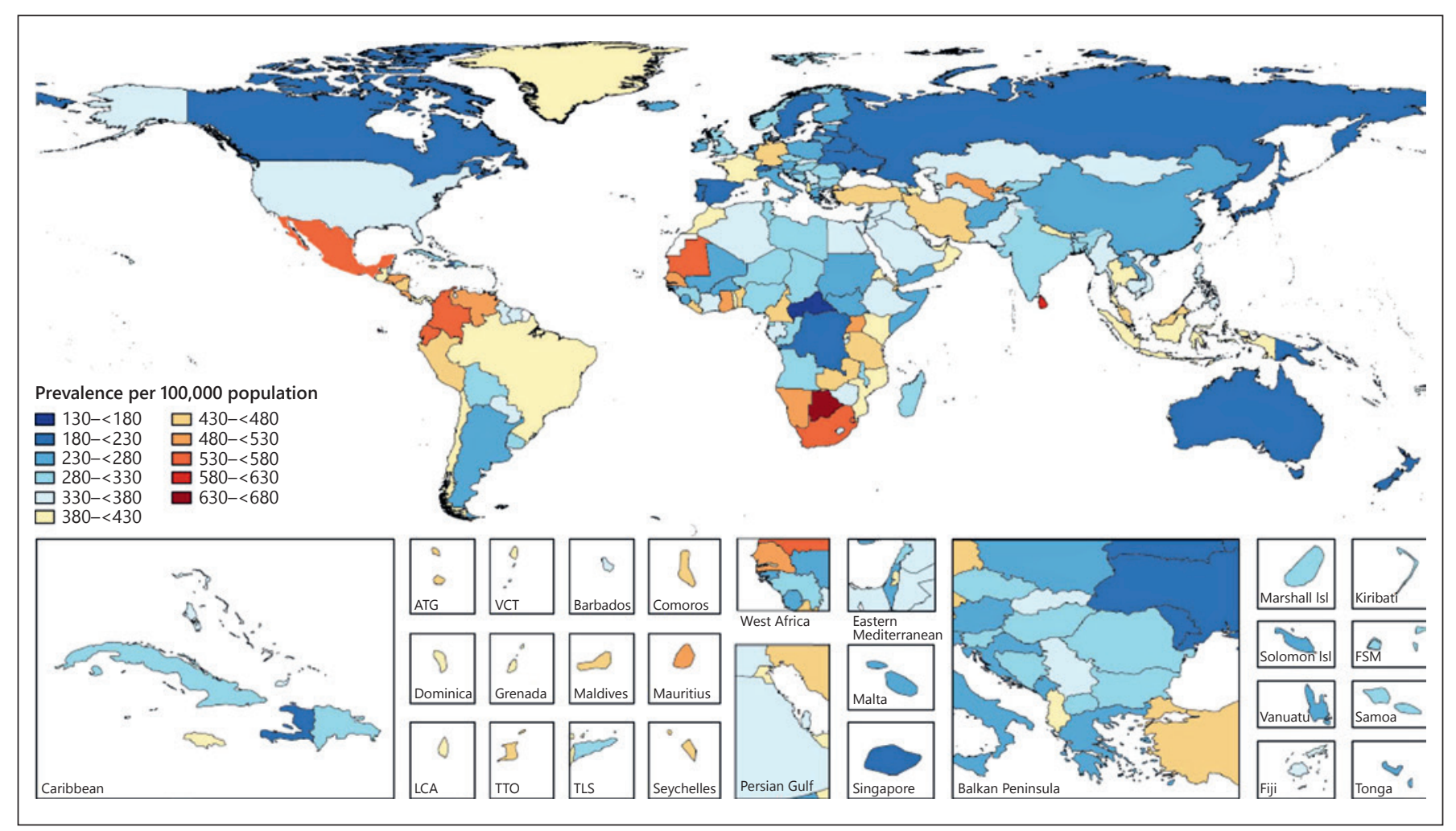

Fig. 1. Age-standardized prevalence $(\times 100,000)$ of idiopathic epilepsy by country, 2016. With permission from Global Burden of Disease 2016 Epilepsy Collaborators [18].

adults, and persons with idiopathic and symptomatic seizures [38]. As with prevalence and incidence, epilepsy mortality reflects the quality of case ascertainment, the accuracy of the information on causes of death and the survey methods [6]. People with epilepsy are at an increased risk of death than the general population [38]. Among deaths attributable to epilepsy or seizures, important immediate causes include SUDEP, SE, unintentional injuries, and suicide.

In HIC, standardized mortality ratio ranges from 1.6 to 3.0 [38]. In LMIC, the corresponding ratio is 19.8 (95\% CI 9.7-45.1) [39]. Standardized mortality ratio is slightly higher in men than in women and in children and adolescents, in people with epilepsies due to documented etiology, and in those reporting less adherence to treatment. Indirect causes of death in LMIC include not only drowning and burns but also lack of access to health facilities and preventable causes.

The incidence of SUDEP among people with epilepsy is 1.2 per 1,000 person-years (95\% CI $0.9-1.5$ ) and ranges from 1.1 (95\% CI 0.5-2.3) in children under age 16 years to 1.3 (95\% CI $0.9-1.8$ ) in adults after the age of 50 years [40]. The major risk factors include generalized tonic- clonic seizures, nocturnal seizures, and persistence of seizures. Freedom from seizures, particularly generalized tonic-clonic, is associated with decreased risk and nocturnal supervision is protective [41].

\section{Burden of Epilepsy}

According to the 2016 Global Burden of Disease Collaborators [18], epilepsy represents a relevant fraction of the worldwide disease burden, accounting for about 46 million people. Nearly $80 \%$ of people with epilepsy reside in LMIC, where rates of epilepsy prevalence and incidence are higher than in HIC [42]. The differences are likely due to differing causes, a higher incidence of injuries, and lack of access to health care.

In 2016, epilepsy accounted for $>13$ million DALYs and was responsible for $0.5 \%$ of the total disease burden [18]. In terms of age-standardized DALY rates for all neurological disorders by Global Burden of Disease region in 2016, epilepsy ranked second to eighth depending on the geographic region. The burden of idiopathic epilepsy 
(i.e., due to a genetic cause or when diagnostic assessment did not reveal a causative factor) was highest in eastern, western, and southern sub-Saharan Africa, central Asia, central and Andean Latin America, and southeast Asia (Fig. 1).

Age-standardized DALYs were 182.6 per 100,000 population, 163.6 per 100,000 population for women, and 201.2 per 100,000 population for men. The higher DALY rates in men than in women were due to higher years of life lost rates. Between 1990 and 2016, there was a nonsignificant $6.0 \%$ increase in the age-standardized prevalence of idiopathic epilepsy, but a significant decrease in agestandardized mortality rates $(-24.5 \%)$ and age-standardized DALY rates $(-19.4 \%)$.

Between 1990 and 2016, a significant reduction was observed in the mortality rate in people with idiopathic epilepsy and, to a lesser extent, a reduction was found in DALY rates [18]. This finding probably reflects improvements in access to health facilities and treatment, which in turn may lead to a lesser severity of the disease and lower risk of death.

\section{The Change of the Population Health and Its Impact on the Epidemiology of Epilepsy}

The data on the global burden of epilepsy have clearly demonstrated that, contrary to other clinical conditions, the disease presents a decreasing trend, mostly explained by a significant reduction of mortality. A significant decrease of communicable diseases as a reflection of better sanitation and the introduction of preventive measures leads to the reduction of many environmental risk factors and epileptogenic conditions. Better health care is also followed by increased survival and, in turn, by a longer life expectancy. The progressive aging of the worldwide population is accompanied by a shift of age-specific incidence and prevalence of epilepsy with a progressive decrease of the disease in the youngest age-groups and a corresponding increase in the elderly. A better control of preventable causes of epileptic seizures, which mostly include pre-/perinatal injuries, CNS infections and infestations, TBI, and stroke [43], will be followed by a decrease of these clinical conditions but an increase in aging-related diseases (in particular, CNS tumors and Alzheimer disease and other dementias).

\section{Conclusions and Future Directions}

Despite the decrease in the disease burden, epilepsy is still an important cause of disability and mortality. If applied to epidemiological studies, the change in the definition of epilepsy, which now includes a significant number of cases with single unprovoked seizures, will affect incidence, prevalence, and mortality of epilepsy in the future. However, the collection of data on epilepsy in representative population samples and using standardized methods will strengthen the estimates and will provide accurate findings in countries for which we currently have no or sparse data and if additional data are collected on severity, causes, and treatments. Sizable gains in reducing the burden of epilepsy might be expected from improved access to existing treatments in low-income countries and from the development of new effective drugs worldwide.

\section{Disclosure Statement}

Dr. Ettore Beghi reports grants from UCB-Pharma, SOBI and the Italian Ministry of Health.

\section{References}

1 Fisher RS, van Emde Boas W, Blume W, Elger C, Genton P, Lee P, et al. Epileptic seizures and epilepsy: definitions proposed by the International League Against Epilepsy (ILAE) and the International Bureau for Epilepsy (IBE). Epilepsia. 2005 Apr;46(4):470-2.

2 Neligan A, Hauser WA, Sander JW. The epidemiology of the epilepsies. Handb Clin Neurol. 2012;107:113-33.

3 Beghi E, Carpio A, Forsgren L, Hesdorffer DC, Malmgren K, Sander JW, et al. Recommendation for a definition of acute symptomatic seizure. Epilepsia. 2010 Apr;51(4):671-5.

4 Hesdorffer DC, Benn EK, Cascino GD, Hauser WA. Is a first acute symptomatic seizure epilepsy? Mortality and risk for recurrent seizure. Epilepsia. 2009 May;50(5):1102-8.

5 Fisher RS, Acevedo C, Arzimanoglou A, Bogacz A, Cross JH, Elger CE, et al. ILAE official report: a practical clinical definition of epilepsy. Epilepsia. 2014 Apr;55(4):475-82.

6 Thurman DJ, Beghi E, Begley CE, Berg AT, Buchhalter JR, Ding D, et al.; ILAE Commission on Epidemiology. Standards for epidemiologic studies and surveillance of epilepsy. Epilepsia. 2011 Sep;52 Suppl 7:2-26.

7 Fisher RS, Cross JH, French JA, Higurashi N, Hirsch E, Jansen FE, et al. Operational classification of seizure types by the International League Against Epilepsy: Position Paper of the
ILAE Commission for Classification and Terminology. Epilepsia. 2017 Apr;58(4):522-30.

8 Trinka E, Cock H, Hesdorffer D, Rossetti AO, Scheffer IE, Shinnar S, et al. A definition and classification of status epilepticus - Report of the ILAE Task Force on Classification of Status Epilepticus. Epilepsia. 2015 Oct;56(10): 1515-23.

9 Nashef L, So EL, Ryvlin P, Tomson T. Unifying the definitions of sudden unexpected death in epilepsy. Epilepsia. 2012 Feb;53(2): 227-33.

10 Hauser WA, Beghi E. First seizure definitions and worldwide incidence and mortality. Epilepsia. 2008;49 Suppl 1:8-12. 
11 Fiest KM, Sauro KM, Wiebe S, Patten SB, Kwon CS, Dykeman J, et al. Prevalence and incidence of epilepsy: A systematic review and meta-analysis of international studies. Neurology. 2017 Jan;88(3):296-303.

12 Beghi E, Hesdorffer D. Prevalence of epilepsy - an unknown quantity. Epilepsia. 2014 Jul; 55(7):963-7.

13 Kelvin EA, Hesdorffer DC, Bagiella E, Andrews H, Pedley TA, Shih TT, et al. Prevalence of self-reported epilepsy in a multiracial and multiethnic community in New York City. Epilepsy Res. 2007 Dec;77(2-3):141-50.

14 Kaiboriboon K, Bakaki PM, Lhatoo SD, Koroukian S. Incidence and prevalence of treated epilepsy among poor health and low-income Americans. Neurology. 2013 May; 80(21):1942-9.

15 Bharucha NE, Bharucha EP, Bharucha AE, Bhise AV, Schoenberg BS. Prevalence of epilepsy in the Parsi community of Bombay. Epilepsia. 1988 Mar-Apr;29(2):111-5.

16 Hauser WA, Annegers JF, Kurland LT. Incidence of epilepsy and unprovoked seizures in Rochester, Minnesota: 1935-1984. Epilepsia. 1993 May-Jun;34(3):453-68.

17 Camfield P, Camfield C. Incidence, prevalence and aetiology of seizures and epilepsy in children. Epileptic Disord. 2015 Jun;17(2): $117-23$.

18 GBD 2016 Neurology Collaborators. Global, regional, and national burden of neurological disorders, 1990-2016: a systematic analysis for the Global Burden of Disease Study 2016. Lancet Neurol. 2019;18:357-75.

19 Wirrell EC, Grossardt BR, Wong-Kisiel LC, Nickels KC. Incidence and classification of new-onset epilepsy and epilepsy syndromes in children in Olmsted County, Minnesota from 1980 to 2004: a population-based study. Epilepsy Res. 2011 Jun;95(1-2):110-8.

20 Senanayake N, Román GC. Epidemiology of epilepsy in developing countries. Bull World Health Organ. 1993;71(2):247-58.

21 Chin RF, Neville BG, Scott RC. A systematic review of the epidemiology of status epilepticus. Eur J Neurol. 2004 Dec;11(12):800-10.

22 Zarrelli MM, Beghi E, Rocca WA, Hauser WA. Incidence of epileptic syndromes in Rochester, Minnesota: 1980-1984. Epilepsia. 1999 Dec;40(12):1708-14.

23 Scheffer IE, Berkovic S, Capovilla G, Connolly MB, French J, Guilhoto L, et al. ILAE clas- sification of the epilepsies: Position paper of the ILAE Commission for Classification and Terminology. Epilepsia. 2017 Apr;58(4):51221.

24 Giussani G, Franchi C, Messina P, Nobili A, Beghi E; EPIRES Group. Prevalence and incidence of epilepsy in a well-defined population of Northern Italy. Epilepsia. 2014 Oct;55(10): 1526-33.

25 Annegers JF, Hauser WA, Elveback LR. Remission of seizures and relapse in patients with epilepsy. Epilepsia. 1979 Dec;20(6):72937.

26 Sillanpää M, Schmidt D. Natural history of treated childhood-onset epilepsy: prospective, long-term population-based study. Brain. 2006 Mar;129(Pt 3):617-24.

27 Beghi E, Giussani G, Sander JW. The natural history and prognosis of epilepsy. Epileptic Disord. 2015 Sep;17(3):243-53.

28 Brodie MJ, Barry SJ, Bamagous GA, Norrie JD, Kwan P. Patterns of treatment response in newly diagnosed epilepsy. Neurology. 2012 May;78(20):1548-54.

29 Shorvon SD, Goodridge DM. Longitudinal cohort studies of the prognosis of epilepsy: contribution of the National General Practice Study of Epilepsy and other studies. Brain. 2013 Nov;136(Pt 11):3497-510.

30 Berg AT, Rychlik K. The course of childhoodonset epilepsy over the first two decades: a prospective, longitudinal study. Epilepsia. 2015 Jan;56(1):40-8.

31 Giussani G, Canelli V, Bianchi E, Erba G, Franchi C, Nobili A, et al.; EPIRES Group. Long-term prognosis of epilepsy, prognostic patterns and drug resistance: a populationbased study. Eur J Neurol. 2016 Jul;23(7): 1218-27.

32 Berg AT, Shinnar S. The risk of seizure recurrence following a first unprovoked seizure: a quantitative review. Neurology. 1991 Jul; 41(7):965-72.

33 Mbuba CK, Ngugi AK, Newton CR, Carter JA. The epilepsy treatment gap in developing countries: a systematic review of the magnitude, causes, and intervention strategies. Epilepsia. 2008 Sep;49(9):1491-503.

34 Placencia M, Shorvon SD, Paredes V, Bimos C, Sander JW, Suarez J, et al. Epileptic seizures in an Andean region of Ecuador. Incidence and prevalence and regional variation. Brain. 1992 Jun;115(Pt 3):771-82.
35 Watts AE. The natural history of untreated epilepsy in a rural community in Africa. Epilepsia. 1992 May-Jun;33(3):464-8.

36 Nicoletti A, Sofia V, Vitale G, Bonelli SI, Bejarano V, Bartalesi F, et al. Natural history and mortality of chronic epilepsy in an untreated population of rural Bolivia: a follow-up after 10 years. Epilepsia. 2009 Oct;50(10):2199 206.

37 Sander JW. Some aspects of prognosis in the epilepsies: a review. Epilepsia. 1993 Nov-Dec; 34(6):1007-16.

38 Thurman DJ, Logroscino G, Beghi E, Hauser WA, Hesdorffer DC, Newton CR, et al.; Epidemiology Commission of the International League Against Epilepsy. The burden of premature mortality of epilepsy in high-income countries: A systematic review from the Mortality Task Force of the International League Against Epilepsy. Epilepsia. 2017 Jan;58(1): $17-26$.

39 Levira F, Thurman DJ, Sander JW, Hauser WA, Hesdorffer DC, Masanja H, et al.; Epidemiology Commission of the International League Against Epilepsy. Premature mortality of epilepsy in low- and middle-income countries: A systematic review from the Mortality Task Force of the International League Against Epilepsy. Epilepsia. 2017 Jan;58(1): 6-16.

40 Sveinsson O, Andersson T, Carlsson S, Tomson $T$. The incidence of SUDEP: A nationwide population-based cohort study. Neurology. 2017 Jul;89(2):170-7.

41 Harden C, Tomson T, Gloss D, Buchhalter J, Cross JH, Donner E, et al. Practice guideline summary: Sudden unexpected death in epilepsy incidence rates and risk factors: Report of the Guideline Development, Dissemination, and Implementation Subcommittee of the American Academy of Neurology and the American Epilepsy Society. Neurology. 2017 Apr;88(17):1674-80.

42 Meyer AC, Dua T, Ma J, Saxena S, Birbeck G. Global disparities in the epilepsy treatment gap: a systematic review. Bull World Health Organ. 2010 Apr;88(4):260-6.

43 Thurman DJ, Begley CE, Carpio A, Helmers $\mathrm{S}$, Hesdorffer DC, Mu J, et al. The primary prevention of epilepsy: A report of the Prevention Task Force of the International League Against Epilepsy. Epilepsia. 2018 May;59(5):905-14. 